Sigstad et $a l^{1}$ conclusion, that this panel of markers is not valid to distinguish FTA and FTC, was also based on Matsumoto et al ${ }^{6}$ data. However, in their manuscript, C1orf24 was expressed in nearly all PTC and 50\% of FTC, whereas was not expressed in normal thyroid. Notably, we have also emphasized that none of these markers should be used alone.

We also share with other groups a strong belief that C1orf24 has a role in the pathogenesis of other tumor subtypes. Ito et $a l^{7}$ found that C1orf24 was positive in $97 \%$ of head and neck squamous cell carcinoma and in $66 \%$ of dysplastic lesion, whereas was not observed in normal mucosal samples. It was also identified as a new candidate marker for renal carcinogenesis, as it was positive in sporadic human renal carcinoma cell lines and not observed in normal kidney. ${ }^{5}$ Finally, functional analyses have supported that C1orf24 have a potential role in protecting cells from genotoxic stress-induced apoptosis. ${ }^{8}$ In addition, they demonstrated that C1orf24 depletion increased cell apoptosis. The authors suggest that AKT contributes to resistance of cancer cells through C1orf24 phosphorylationpromoted P53 degradation. ${ }^{8}$

It is clear that there is a need to perform more studies using this panel of markers. A practical challenge to demonstrate the clinical application of our proposed antibody-based test is that we had used custom-produced antibodies. Currently, however, there are few companies that generated antibodies against ITM1, C1orf24 and PVALB. Unquestionably, a study designed to compare antibodies of various sources, including all commercially available and custom produced, should be performed to determine antibodies specificity and reproducibility and, therefore, whether they are useful markers that can be applied into clinical practice. However, one should have in mind that one batch of an antibody may work, whereas the next, with a different lot, may not. As antibodybased test will be widely used on clinical decisions, standardization and development of antibody guidelines are critical. For the meantime, if of our responsibility to have our eyes open to all potential problems and assure that the results are replicable and likely to be correct.

\section{Disclosure/conflict of interest}

The authors declare no conflict of interest.

Gianna M Carvalheira, Bruno HN Nozima, Gregory J Riggins and Janete M Cerutti Laboratório a Base Genética dos Turmores da Tiroide, Pedro de Toledo 669, 11 andar, Department of Morphology and Genetics, Federal University of São Paulo, 04039-032, São Paulo, SP, Brazil E-mail: j.cerutti@unifesp.br

\section{References}

1 Sigstad E, Paus E, Bjoro T, et al. The new molecular markers DDIT3, STT3A, ARG2 and FAM129A are not useful in diagnosing thyroid follicular tumors. Mod Pathol 2012;25:537-547.

2 Cerutti JM, Delcelo R, Amadei MJ, et al. A preoperative diagnostic test that distinguishes benign from malignant thyroid carcinoma based on gene expression. J Clin Invest 2004;113:1234-1242.

3 Cerutti JM, Latini FR, Nakabashi C, et al. Diagnosis of suspicious thyroid nodules using four protein biomarkers. Clin Cancer Res 2006;12:3311-3318.

4 Cerutti JM, Oler G, Delcelo R, et al. PVALB, a new Hurthle adenoma diagnostic marker identified through gene expression. J Clin Endocrinol Metab 2011;96:E151-E160.

5 Adachi $\mathrm{H}$, Majima S, Kon S, et al. Niban gene is commonly expressed in the renal tumors: a new candidate marker for renal carcinogenesis. Oncogene 2004;23:3495-3500.

6 Matsumoto F, Fujii H, Abe M, et al. A novel tumor marker Niban, is expressed in subsets of thyroid tumors and Hashimoto's thyroiditis. Hum Pathol 2006;37:1592-1600.

7 Ito S, Fujii H, Matsumoto T, et al. Frequent expression of Niban in head and neck squamous cell carcinoma and squamous dysplasia. Head Neck 2010;32:96-103.

8 Ji H, Ding Z, Hawke D, et al. AKT-dependent phosphorylation of Niban regulates nucleophosmin- and MDM2-mediated p53 stability and cell apoptosis. EMBO Rep 2012;13:554-560.

\title{
Reply to 'The new molecular markers DDIT3, STT3A, ARG2 and FAM129A are not useful in diagnosing thyroid follicular tumors'
}

\author{
Modern Pathology (2013) 26, 613-615; doi:10.1038/modpathol.2013.39
}

To the Editor: In reply to Carvalheira et al's ${ }^{1}$ letter regarding our article, ${ }^{2}$ we would like to answer as follows:

First, considering the urgent need for markers to distinguish between follicular adenoma (FTA) and carcinoma (FTC) during FNA cytology, we find it remarkable that no clinical studies have been published validating the four classifiers originally described by Cerutti et al. ${ }^{3}$ Indeed, we are unaware of any diagnostic laboratory using these markers 
even though they were originally estimated to have a sensitivity of 1.00 for detecting malignancy.

In their letter, Carvalheira et al. ${ }^{1}$ express a concern regarding the specificity of the custom polyclonal antibodies we raised against FAM129A and STT3A. Readers of Modern Pathology, and especially those who use IHC techniques in the diagnostic laboratory, are well aware of the thorny issues that can arise when assessing antibody specificity. In this regard, two of us (DJW and EP) are participants in the International Society of Oncology and Biomarkers (ISOBM) TD-workshops, which since 1996 have characterized the epitope structure and specificities of $>350$ diagnostically relevant monoclonal antibodies. ${ }^{4}$

Our anti-FAM129A and -STT3A were raised using synthetic peptides identical to those used by Cerutti et $a l^{3}$ Although results from peptide-blocking studies must be evaluated with caution, ${ }^{5}$ the ability of unconjugated peptide to abrogate tissue staining strongly supported the anti-peptide specificity of our reagents. It was during western blotting experiments (to assess off-target binding) that we found significant expression of ARG2, STT3A and FAM129A in many FTA lysates. Cerutti et $a .^{3}$ also characterized their anti-FAM129A and anti-STT3A by western blotting. However, although they included lysates from papillary thyroid carcinoma, FTC and normal thyroid, they failed to include any FTA tissue. This seems a remarkable omission considering they were developing reagents to discriminate FTA from FTC.

In their letter, Carvalheira et al. suggest that the absence of a 70-kD band in our FAM129A western blots indicates that our antiserum has a different reactivity to the reagent they used. We refer them to Figure 5 where a band of this size can be seen in lanes 1, 2 and 7. In agreement with their observations, we found some tissues (lane 8) that only display the 70-kD FAM129A fragment. If the 70-kD band is indeed a 'stress marker', ${ }^{6}$ we probably failed to detect it in the FTC-133 cell line simply because we prepare lysates from log-phase cells. Do Carvalheira et al. use confluent 'stressed' cultures?

In regard to the somewhat smaller band seen in our anti-STT3A western blots, discrepancies between predicted and measured protein sizes frequently occur. A plethora of factors acting alone, or together, can influence protein mobility in SDSPAGE. Furthermore, during western transfers small errors in the registration of gel/membrane/film can occasionally occur. A quick web-based search for STT3A western blots show reported reactivity from around $60 \mathrm{kD}$ to as high as $90 \mathrm{kD}$.

In their letter, Carvalheira et al. ${ }^{1}$ discuss the specificity of our 'in-house'-generated reagents but avoid mention of the commercial anti-ARG2 and GADD153 antibodies they used. In their original paper, no statement was made about how they validated these commercial reagents. This is surprising and raises some concern since we and others $^{7}$ have found the anti-GADD153 reagent to show very poor selectivity.

In our article we clearly state that we performed extensive staining trials including an assessment of the endogenous peroxidase blocking specifically mentioned by Carvalheira et al. ${ }^{1}$ As the principal aim of the method is to discriminate FTC from FTA, we also performed parallel antibody titrations on serial paraffin sections of both tumor types. In our laboratory, internationally standardized fixatives, time prior to fixation, tissue processing and length of fixation time are used. We handle tissue samples from most organs, utilizing hundreds of commercial antibodies in everyday diagnostics as well as in research projects. New antibodies are introduced into our routine on a regular basis, with a standardized way of implementation. Cases from our laboratory are regularly sent for second opinion to approved laboratories in different countries. These immunostained slides are seldom questioned. We therefore find it highly unlikely that factors concerning fixation/antibody dilution/blocking would be of any significance. Indeed, it is generally agreed that all markers for use in diagnostics ought to be robust. A marker is of no practical use if even small differences in, for example, fixation time will have a significant effect on the result.

In conclusion, our peptide-blocking, western and IHC data clearly demonstrate that our custom reagents show good specificity and that STT3A, ARG2 and FAM129A are expressed in a significant number of follicular adenomas. Data from western blotting and IHC studies are concordant even though the tissues are processed in a very different manner prior to analysis.

Thus, we believe that the suggestions made by Carvalheira et al. do not abrogate the conclusion that DDIT3, STT3A, ARG2 and FAM129A are not clinically useful in distinguishing between follicular thyroid adenoma and follicular thyroid carcinoma.

\section{Disclosure/conflict of interest}

The authors declare no conflict of interest.

Eva Sigstad $^{1}$, Elisabeth Paus ${ }^{2}$, Trine Bjøro ${ }^{2,3}$, Aasmund Berner ${ }^{1,3}$, Krystyna Kotanska Grøholt ${ }^{1}$, Lars H Jørgensen ${ }^{4}$, Manuel Sobrinho-Simões ${ }^{5}$, Ruth Holm ${ }^{1}$ and David J Warren ${ }^{2}$ ${ }^{1}$ Division of Diagnostics and Intervention, Department of Pathology, Oslo University Hospital HF, Montebello, Norway; ${ }^{2}$ Division of Diagnostics and Intervention, Department of Medical

Biochemistry, Oslo University Hospital HF, Montebello, Norway; ${ }^{3}$ Institute of Clinical Medicine, University of Oslo, Oslo, Norway; ${ }^{4}$ Division of Cardiovascular and Pulmonary Diseases, Department of Thoracic Surgery, Oslo University Hospital HF, Montebello, Norway; 


\author{
${ }^{5}$ IPATIMUP-Institute of Molecular \\ Pathology and Immunology, University of Porto, \\ Porto, Portugal \\ E-mail: ESP@ous-hf.no
}

\section{References}

1 Carvalheira GM, Nozima BHN, Riggins GJ, et al. DDIT3, STT3A (ITM1), ARG2 and FAM129A (Niban, C1orf24) in diagnosing thyroid carcinoma: variables that may affect the performance of this antibody-based test and promise. Mod Pathol 2013;26:611-613.

2 Sigstad E, Paus E, Bjoro T, et al. The new molecular markers DDIT3, STT3A, ARG2 and FAM129A are not useful in diagnosing thyroid follicular tumors. Mod Pathol 2012;25:537-547.
3 Cerutti JM, Latini FR, Nakabashi C, et al. Diagnosis of suspicious thyroid nodules using four protein biomarkers. Clin Cancer Res 2006;12:3311-3318.

4 Rye PD, Nustad K, Stigbrand T. Tumor marker workshops. Tumour Biol 2003;24:165-171.

5 Willingham MC. Conditional epitopes. Is your antibody always specific? J Histochem Cytochem 1999;47: 1233-1236.

6 Adachi $\mathrm{H}$, Majima S, Kon S, et al. Niban gene is commonly expressed in the renal tumors: a new candidate marker for renal carcinogenesis. Oncogene 2004;23:3495-3500.

7 Haataja L, Gurlo T, Huang CJ, et al. Many commercially available antibodies for detection of CHOP expression as a marker of endoplasmic reticulum stress fail specificity evaluation. Cell Biochem Biophys 2008; 51:105-107.

\section{Nested melanoma}

Modern Pathology (2013) 26, 615-616; doi:10.1038/modpathol.2012.219

To the editor: The recent paper by Heinz Kutzner and co-authors introduces a new variant of superficial spreading melanoma composed predominantly of large hypercellular nests of melanocytes. ${ }^{1}$ The recognition of this particular pattern without a pagetoid element has been delayed as the histopathology masquerades as an unusual nevus. The clinical features, dermoscopy, the distinctive histopathology and now the application of comparative genomic hybridization, all help to identify this as a melanoma variant. Although the authors have linked this unusual nevoid melanoma to superficial spreading melanoma, this has necessitated a major change in the histopathological criteria traditionally applied to superficial spreading melanoma. There may be alternative distinctive pathways that need to be considered.

While exploring the relationship of atypical (dysplastic) nevi in the elderly to melanoma, ${ }^{2}$ the same experience emerged in the discrepancy between the clinical and dermoscopic findings that favoured a melanoma and the histopathological features which appeared nevoid. These melanocytic lesions when broad-based,often had criteria for defining transition to lentigo maligna ${ }^{3}$ or lentiginous melanoma. ${ }^{4}$ More recently, the results of FISH analysis have supported the existence of this controversial subtype of lentiginous melanoma ${ }^{5}$ that often evolves from atypical lentiginous nevi.

The atypical nevus of the elderly is an unstable nevus, and one variation which I have also observed $^{2}$ is the hypercellular nested variant described by the authors. I have reported these as in situ nevoid melanomas. The cellular morphology is usually associated with crowded small to medium hyperchromatic melanocytes. The progression of these atypical nested melanomas is often to a small cell (nevoid) melanoma, which may become desmoplastic.

Although from a clinical perspective the term superficial spreading melanoma is appropriate for this nested melanoma, at least some of these tumors may be linked to an aberrant nevus pathway seen in elderly individuals explaining their unusual pattern that resembles a bizarre nevus.

The authors have clearly defined and have highlighted a potentially important variant of melanoma that will now be recognized more frequently. The eventual classification of this melanoma needs further work in reference to the unstable atypical nevi seen in elderly individuals that may evolve as melanomas including this predominantly nested non lentiginous pathway.

\section{Disclosure/conflict of interest}

The authors declare no conflict of interest.

Steven Kossard
Department of Dermatopathology, Skin \& Cancer
Foundation Australia, Sydney, NSW, Australia
E-mail: skossard@scfa.edu.au

\section{References}

1 Kutzner H, Metzler G, Argenyi Z, et al. Histological and genetic evidence for a variant of superficial spreading melanoma composed predominantly of large nests. Mod Pathol 2012;25:838-845.

2 Kossard S. Atypical lentiginous junctional naevi of the elderly and melanoma. Australas J Dermatol 2002; 43:93-101. 